\title{
Dix commandements mais pas de Dieu : christianisme et changement socioculturel chez les Baruya de Papouasie Nouvelle-Guinée
}

The ten Commandments but no God: Christianity and Socio-cultural Change

among thee Baruya of Papua New Guinea

\section{Anne-Sylvie Malbrancke}

\section{(2) OpenEdition}

\section{Journals}

\section{Édition électronique}

URL : http://journals.openedition.org/jso/7502

DOI : $10.4000 /$ jso. 7502

ISSN : $1760-7256$

Éditeur

Société des océanistes

\section{Édition imprimée}

Date de publication : 31 décembre 2016

Pagination : 241-256

ISSN : 0300-953x

\section{Référence électronique}

Anne-Sylvie Malbrancke, « Dix commandements mais pas de Dieu : christianisme et changement socioculturel chez les Baruya de Papouasie Nouvelle-Guinée », Journal de la Société des Océanistes [En ligne], 142-143 | 2016, mis en ligne le 31 décembre 2018, consulté le 10 décembre 2020. URL : http:// journals.openedition.org/jso/7502 ; DOI : https://doi.org/10.4000/jso.7502

\section{(c) (†) $\ominus$}

Journal de la société des océanistes est mis à disposition selon les termes de la Licence Creative Commons Attribution - Pas d'Utilisation Commerciale - Pas de Modification 4.0 International. 


\section{Dix commandements mais pas de Dieu : christia- nisme et changement socioculturel chez les Baruya (PNG)}

par

Anne-Sylvie MALBRANCKE*

\section{RÉSUMÉ}

L'anthropologie du christianisme en Mélanésie a récemment permis d'enrichir les théories du changement social en prônant une approche centrée principalement autour $d u$ concept de rupture. Riche analytiquement, cette perspective doit cependant être nuancée pour envisager des situations où le christianisme est un idiome qui, loin de rompre avec des valeurs morales indigènes, les renforce. Parmi les Baruya de Nouvelle-Guinée, une perception indigène séculière de la religion chrétienne supplante et informe le contenu théologique chrétien, qui ne transforme pas radicalement la hiérarchie locale des valeurs morales. Ce sont des orientations traditionnelles relatives aux relations entre les sexes et à la stratification sociale qui informent l'utilisation de métaphores religieuses, employées comme justification moderne de comportements traditionnels. Le concept de structure de conjonctures permet d'articuler les modèles de rupture et ceux prônant la continuité dans le changement.

Mots-CLÉs : Mélanésie, Papouasie Nouvelle-Guinée, anthropologie du christianisme, changement social, relations entre les sexes, structure des conjonctures

«Moïse a reçu les Dix Commandements. Or nous les Baruya, on obéissait déjà à ce type de lois. Les règles de l'Église sont les mêmes que celles de notre coutume; tu ne dois pas voler, coucher avec la femme d'un autre homme. Tu dois rester sur tes terres, sans aller du côté de la rivière qui appartient à un autre homme. L'Église est arrivée, et on a d'abord cru qu'elle nous raconterait des mythes différents des nôtres, mais on a entendu ses règles et ce sont les mêmes que les Dix Lois des Baruya. »

\section{ABSTRACT}

The anthropology of Christianity in Melanesia has recently expanded and refined models of social change, promoting the concept of rupture as a principal analytical lens. This perspective begs greater nuance for circumstances in which Christian idioms and contents are used to justify rather than contravene or transform long standing non-Christian values and practices. Among the Baruya of Papua New Guinea, an indigenously secularized approach to Christianity supplants and informs Christian theological content without radically altering or modifying the traditional hierarchy of moral values. Instead, indigenous values regarding gender relations and social stratification orient the use of religious metaphors, which justify and sometimes reinforce traditional behaviors while casting them as modern. Analytically, Sahlins' concept of "the structure of conjuncture" allows to articulate models of ruptural Christianity with those of continuity within change.

Keywords: Melanesia, Papua New Guinea, anthropology of Christianity, social change, gender relations, structure of conjuncture

Voilà ce que me disait Tsimion, un Baruya d'une quarantaine d'années, un jour de juillet 2013 où nous nous entretenions au village de Wuyabo (Eastern Highlands, Papouasie Nouvelle-Guinée). Plus de 40 ans après Maurice Godelier, je séjournai pour 12 mois dans la vallée de Wonenara, avec ceux qui avaient été ses amis et interlocuteurs et leurs descendants ${ }^{1}$. Cette

1. Je souhaite remercier très chaleureusement Laurent Dousset et Bruce Knauft, dont les relectures et commentaires ont été indispensables pour construire cet article. Je remercie également les relecteurs anonymes du JSO, dont les suggestions ont grandement aidé à renforcer l'argumentation. Toutes les maladresses et lacunes restent bien sûr de mon fait. Ce travail a été

* Aix-Marseille Université, CNRs, EHEss, Centre de recherche et de documentation sur l'Océanie, Marseille annesylvie. malbrancke@gmail.com 
ethnographie se penchait principalement sur les mutations de la sphère matrimoniale, notamment suite à l'introduction du prix de la fiancée dans un système autrefois strictement limité à l'échange des femmes. Cet angle d'approche permit d'interroger les changements socio-culturels plus largement, notamment la reconfiguration des relations entre les sexes et le rôle de l'argent au quotidien. Dans cette optique, une de mes interrogations concernait le rapport des individus au christianisme, arrivé chez les Baruya dans les années 1960 (Lemonnier, 2013 : 193-198). Lors de conversations ou d'entretiens avec femmes et hommes de tous âges, je me suis donc plus largement intéressée à la relation que mes contemporains entretenaient avec les différentes confessions présentes dans leur vallée. J'ai été rapidement frappée par la propension locale, illustrée par la citation de Tsimion, à rapprocher les « règles de l'Église " d'un ensemble de règles autochtones exprimées dans les termes de l'idéologie chrétienne. Ce rapprochement de deux logiques culturelles situe les Baruya aux antipodes d'autres groupes du pays où la christianisation aurait remplacé les croyances locales et un ensemble de règles guidant la vie quotidienne. L'exemple le plus connu dans la littérature anthropologique du Pacifique étant celui des Urapmin de Nouvelle-Guinée décrits par Joel Robbins ${ }^{2}$ (e.g. 2004a). Par ce rapprochement, il semble que les Baruya parviennent à concilier intégration de certaines valeurs chrétiennes avec le maintien de valeurs qu'ils considèrent comme endogènes. Dans cette optique, le rapport des Baruya à l'aspect théologique de la religion chrétienne sera éclairant. Je montrerai que leur religion pourrait être qualifiée davantage de civique que de mystique, car il s'agit pour eux d'appliquer un certain nombre de règles situées dans le domaine de la morale, sans se rapporter (le plus souvent) à un contenu théologique impliquant une proximité avec Jésus, le Saint-Esprit, ou d'autres éléments de la doctrine chrétienne.

Regardons rapidement le détail de ces lois telles qu'elles me furent énumérées par un interlocuteur âgé, fervent chrétien et par ailleurs, acteur majeur d'initiations masculines conçues comme d'origine ancestrale. Ces lois relevaient manifestement d'une forme de non-dit, car il me fut difficile d'en obtenir une énumération précise de la part de mes interlocuteurs, qui pourtant se réfé- raient volontiers à l'ensemble des « Dix Lois des Baruya" (en réalité il y en a onze), comme s’il s'agissait d'un donné culturel dont on connaîtrait l'idée générale sans être au fait du détail.

Tu ne convoiteras ni ne voleras la femme d'un autre homme.

Tu ne voleras pas les jardins ou les terres d'un autre homme.

Tu ne tueras aucun homme.

Tu ne violeras aucune jeune fille. Le Soleil te regarde d'en-haut, et te tuera.

Tu ne tueras pas les enfants d'un autre homme.

Tu ne voleras pas les terres non défrichées d'un autre homme.

Tu ne voleras pas le bétel d'un autre homme.

Tu ne voleras pas le pandanus d'un autre homme.

Si les ennemis viennent, tu te montreras fort et tu les tueras.

Si tu tues ton propre sang, tu mourras.

Si un homme arrive de loin, tu devras t'en occuper, l'accueillir et le nourrir. Tu ne peux pas le tuer, tu lui as donné à manger.

Si le rapprochement entre valeurs morales locales et valeurs morales chrétiennes sert de justification à la conversion au christianisme (j'y reviendrai), on remarque en première analyse que ces valeurs appartiennent à des sphères de la vie sociale qui ne sont pas toutes connectées à une forme de spiritualité, qu'elle soit indigène ou chrétienne. Le but de cet article n'est pas de nier qu'il y ait eu conversion chrétienne chez les Baruya. Mais il s'agira d'interroger la nature du contenu religieux retenu par les Baruya et rapproché du leur: en ce sens, je chercherai à démontrer qu'en fait de syncrétisme, c'est in fine la religion chrétienne qui est sécularisée plutôt que le système moral baruya qui est christianisé. Effectivement, non seulement les Baruya retiennent du christianisme un code moral décroché du sacré, mais ils n'en font pas un nouveau "local mode of understanding" (Robbins, 1998 : 301) ; c'est leur façon de voir et de comprendre le monde qui dicte l'adoption de la rhétorique chrétienne, et non l'inverse.

Dans une série de publications majeures pour l'anthropologie du christianisme, Robbins (e.g. 2007a, 2007b, 2010a, 2014) explique que la recherche n’a pas su faire place à une pensée de la rupture : le changement culturel serait toujours

financé par une allocation doctorale de trois ans, versée par l'EHEss Paris, et a été dirigé par Pascale Bonnemère (CREDO), à qui j'adresse mes plus vifs remerciements pour ses conseils et son soutien constant. Ce travail doit beaucoup à Pierre Lemonnier, dont l'aide précieuse m’a été fondamentale pour mener ce terrain.

2. Cette rapide caractérisation ne fait pas justice à la complexité du cas Urapmin décrit par Robbins, où « social structural ideas persist alongside the Christian ideas that have become so important [so] it is fair to describe the Urapmin situation as one in which two distinct cultural logics are in play. Neither of these logics has succeeded in subordinating the other or in reframing the other in its own terms [...]" (2007b: 307). Robbins explique cependant que certaines pratiques apparemment traditionnelles, comme un sacrifice de cochon, sont en réalité inscrites dans une logique chrétienne et subordonnées à une croyance en la salvation par le culte chrétien (2007a : 16). Il explique que seule une analyse de la relation structurant les différentes croyances religieuses permet de déterminer si une culture peut être dite chrétienne. À cet égard je vais tâcher de montrer que la situation des Baruya s'éloigne nettement de celle des Urapmin, dans la mesure où les premiers ne conçoivent pas la relation entre les deux ensembles de valeurs comme étant antagonique. 
peu ou prou vu sous l'angle de l'ancien, de la continuité (voir par exemple le modèle du «develop-man " de Sahlins, 1992), quand le christianisme offre à l'inverse une rupture et l'ouverture vers une dimension où le changement radical est devenu possible (Robbins, 2003 ; Engelke, 2004 ; Keller, 2005). La pensée de la continuité, et la représentation du temps qui l'accompagne, auraient créé un angle mort vis-à-vis du concept de rupture culturelle. Robbins suggère un nouveau cadre d'analyse, qui emprunte à Dumont le concept de " hiérarchie " pour retenir la manière dont les valeurs structurent les relations entre les diverses composantes d'une société. Ce faisant, le modèle de Robbins implique une vision holistique du christianisme, qui est conçu comme un système englobant, un point de non-retour qui transforme et remplace à jamais la culture indigène. Robbins admet certes que le changement puisse se trouver dans d'autres sphères de la société sans nécessairement être impulsé par la conversion au christianisme (2012) : il parle ainsi des Huli des Southern Highlands, étudiés par Holly Wardlow (2006), où les transformations rapides du contexte économique ont, selon cet auteur, eu un impact bien plus profond sur les relations entre les sexes et sur la place des femmes dans le tissu social et les relations de parenté que le christianisme. Cependant cet aspect de son argumentaire reste périphérique. Le présent article se propose d'explorer l'articulation entre rupture et continuité, plutôt que leur antagonisme, en reprenant le concept de "structure des conjonctures » développée par Marshall Sahlins (1981).

\section{Face à un vaste paysage confessionnel : pragmatisme et nomadisme}

Les Baruya de la vallée de Wonenara se trouvent dans une configuration que John Barker, autre spécialiste des recherches sur le christianisme dans le Pacifique, qualifierait de "conversion secondaire " (2012b). Effectivement, cette vallée relativement isolée des Eastern Highlands, accessible à pied ou en avion seulement, fut à deux reprises un front missionnaire (Bonnemère, 2016). Des luthériens arrivèrent en premier dans la vallée au début des années 1960, époque à laquelle ils ouvrirent une école pour enseigner à lire la Bible en pidgin ${ }^{3}$, à écrire et à compter
(Godelier, 1982 : 304). Des Adventistes du Septième Jour (SDA) établirent leur église plus tard chez les voisins et ennemis traditionnels des Baruya, les Ipmani, puis une autre dans le bas de la vallée, près de la station de Wonenara. À la fin des années 1980 et après une guerre de 7 ans entre Baruya et Ipmani, la vallée redevint une "terre de mission" (Lemonnier, 2013: 215) : une Église pentecôtiste, la Church of Christ, apparut en 1987, établie par un Baruya qui découvrit la confession alors qu'il vivait dans une autre province du pays. Elle fut suivie dans les années 2000 de deux autres Églises évangéliques : l'Église pentecôtiste Revival Fellowship, établie en 2002 également par un Baruya ayant découvert le message Revival à Goroka, capitale des Eastern Highlands; enfin, l'Evangelical Brotherhood Church (EBC), une Église protestante de type "évangélique-charismatique", s'implanta en $2008^{4}$ (Lemonnier, 2013 : 201-202). Cette diversité confessionnelle n'est pas un fait rare dans le Pacifique et Jebens parle de "pluralisme dénominationnel " (2011, voir aussi Bonnemère 2016). Les analyses sur le sujet montrent que :

" these new Pentecostal congregations [...] have usually won their adherents from longer established former mission churches. " (Barker, 2001: 105)

Le terrain baruya offre un contraste net entre l'affiliation déclarée des individus et leur fréquentation du culte. Un jour où je me présentais devant les élèves de la grade $8^{5}$ de l'école primaire, trente jeunes hommes et cinq jeunes femmes âgés de 16 à 31 ans, ils acceptèrent de se décrire tour à tour en quelques mots ; spontanément, chacun(e) précisa à quelle Église il ou elle appartenait. Malgré des biais possibles ${ }^{6}$, cette réponse semblait pointer vers une adhésion massive à l'une ou l'autre confession présente dans la vallée. Pourtant, tandis que l'adhésion au christianisme est volontiers déclarée, la fréquentation des différentes Églises reste très marginale. Mes propres observations des différents cultes rejoignent la conclusion de Pierre Lemonnier, qui remarquait lors de sa visite en 2011, qu' « il n'y a pas eu de conversion en masse chez les Baruya, et pour beaucoup d'entre eux les Églises restent un objet de curiosité, comme il y a cinquante ans " (2013 : 215). De fait, en 2013-2014, je fus loin d'observer une assemblée massive - ou régulière - à aucun des cinq offices hebdomadaires de la

3. Les Baruya sont des locuteurs yipma (voir Lloyd 1973) mais parlent aussi tok pisin, langue véhiculaire et langue officielle de Papouasie Nouvelle-Guinée, avec l'anglais et le hiri motu. Je conversais en tok pisin avec la plupart de mes interlocuteurs, ayant recours à un traducteur pour les plus âgé(e)s qui ne parlaient que yipma.

4. Aujourd'hui, comme à son commencement, c'est un pasteur non Baruya qui célèbre l'office, assisté cependant d'un Baruya pour traduire en langue yipma les sermons délivrés en tok pisin.

5. La dernière année du niveau primaire en Papouasie Nouvelle-Guinée.

6. La proximité perçue entre les Blancs et les Églises (car les luthériens présents trente ans chez eux étaient australiens, même si par la suite ce sont des locaux qui ont introduit les confessions plus récentes) les aurait peut-être incités à déclarer une appartenance à une confession ou une autre. 


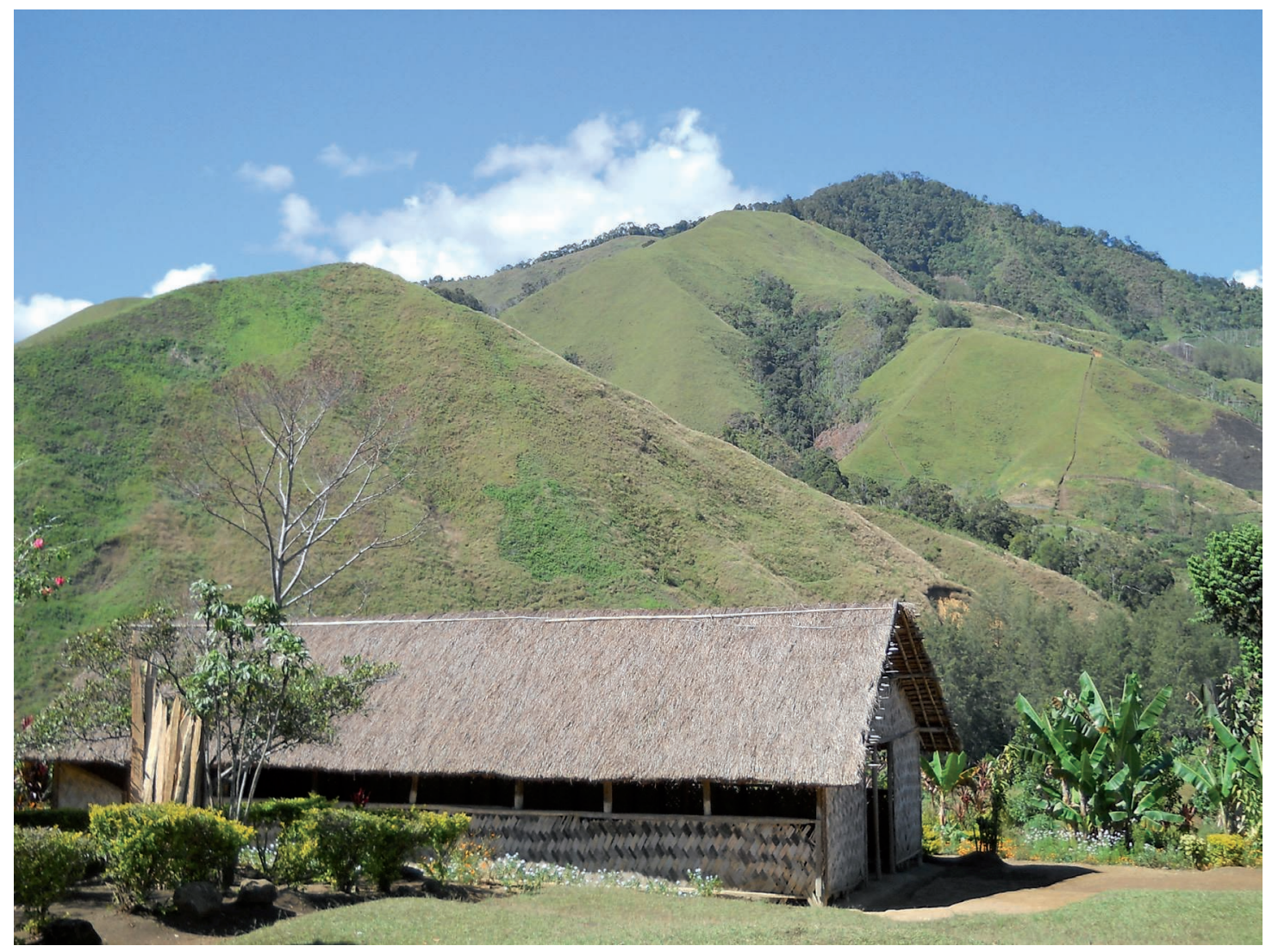

Рното 1. - L'église SDA, vallée de Wonenara, 2013 (@ Anne-Sylvie Malbrancke)

vallée $^{7}$, que l'on appelle "lotu» en tok pisin. Je séjournai au village de Wuyabo, perché en haut de la montagne et accessible par une piste d'atterrissage. Ses 600 habitants en font le village le plus peuplé de la vallée ${ }^{8}$. J'ai dénombré en moyenne une assemblée de 40 personnes ${ }^{9}$ à l'office des luthériens et à celui des EBC, les deux Églises présentes à Wuyabo, et que j'ai pu observer plus souvent. L'Église COC, située à Yanyi, village un peu moins peuplé et situé à 20 minutes de marche en contrebas, attire quant à elle une cinquantaine de personnes chaque semaine ${ }^{10}$. Par contraste, les SDA ainsi que l'Église Revival, dans le bas de la vallée, rassemblent deux à trois fois plus de fidèles, ayant dans leurs rangs des Baruya ainsi que des Ipmani. Alors que la totalité des Baruya de moins de 30 ans, et plus de la majorité des individus plus âgés, me dit se rendre au culte, dans les faits c'est une poignée d'entre eux seulement qui fréquente une église assidûment.

Déserter le culte ne revient pas nécessairement à questionner la place du christianisme, ou même les correspondances établies entre religion exogène et conceptions préchrétiennes de la société et de son cosmos. C'est pourtant la conclusion à laquelle arrive Junius, le pasteur EBC originaire de la même province des Eastern Highlands. Il me confia un jour que selon ses estimations les Baruya fréquentent moins son église que toutes les autres communautés papoues où il a exercé auparavant, et où c'est le village entier qui s'assemble régulièrement au culte. Selon lui, une telle désertion du culte signale un manque d'intérêt local pour la religion chrétienne en général : il déplore qu'on ne lui pose jamais de questions sur l'Eglise ou sur la Bible, qu'on ne le prenne pas à partie dans les affaires du village pour avoir son avis, ou pour obtenir ses conseils et régler un problème. Ni lui ni son Église n'occupent une place quotidienne dans la vie sociale, d'où son sentiment de n'exister "que le jeudi et le dimanche " ${ }^{11}$.

Sans hostilité face au christianisme, les Baruya démontrent un remarquable manque d'intérêt pour les affaires religieuses. Les témoignages

7. L'office EBC a lieu les jeudis et dimanches matin, l'office SDA tous les samedis, et les autres cultes les dimanches (Coc, luthérien, Revival).

8. Je recensai 2426 habitants à Wonenara en 2013.

9. Notons que sur ces 40 , les deux tiers sont des enfants ou des jeunes gens non mariés

10.L'illustration 4 montre que les enterrements chrétiens sont des occasions qui rassemblent davantage de gens, fournissant un moment convivial de commensalité.

11.En 2010, Bonnemère notait que le culte EBC se tenait « les mardis, jeudis, vendredis et dimanches " (2013: 232). Je n'avais pas cette information au moment de mon terrain et n'ai donc pas pu interroger la population ou le pasteur sur cette baisse de fréquence des offices, qui peut être mise sur le compte de la trop faible fréquentation de l'Église dont parle Junius. 


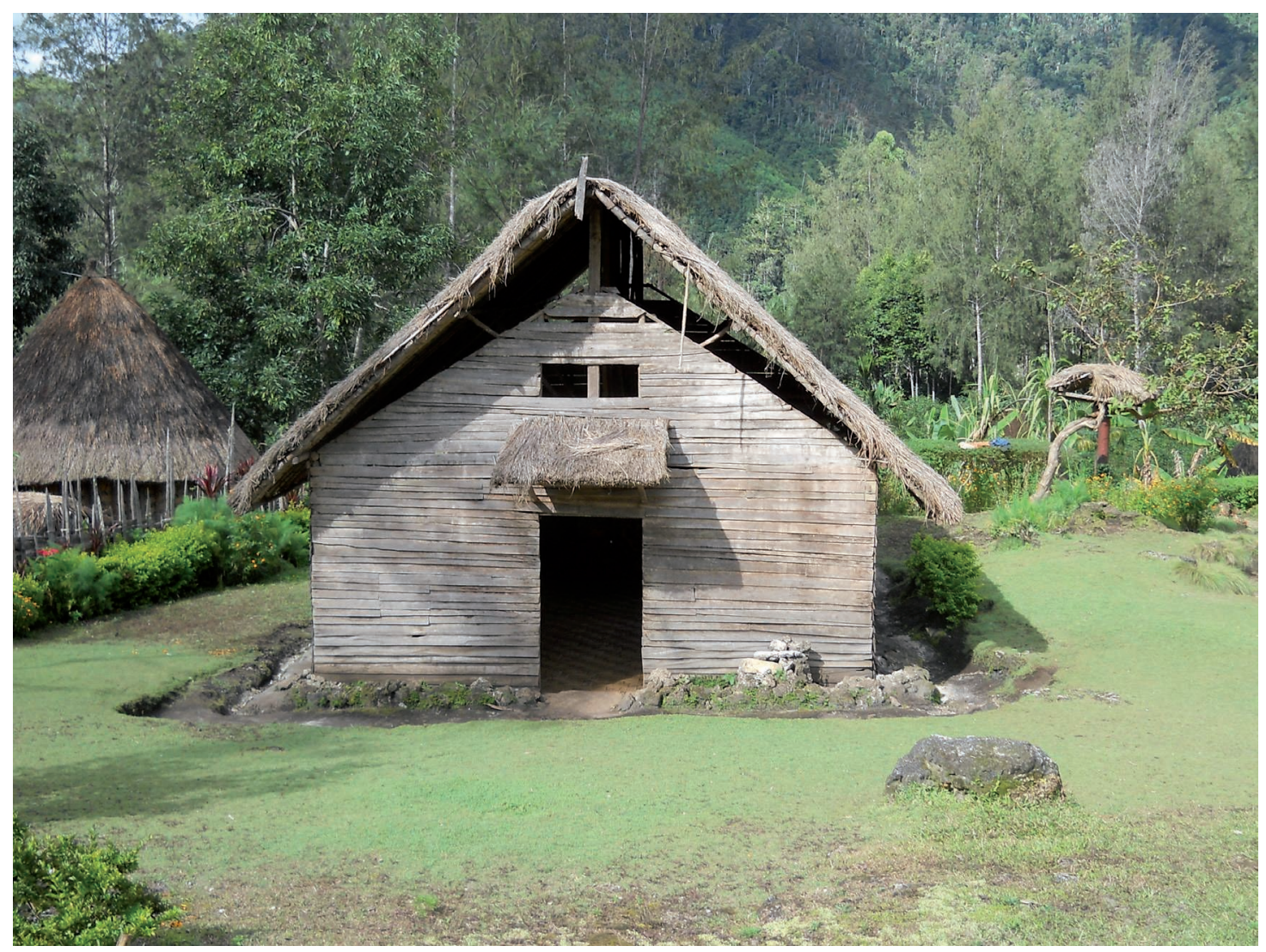

Рното 2. - L'église EвC, village de Wuyabo, vallée de Wonenara, 2013 (@ Anne-Sylvie Malbrancke)

récoltés font état, à quelques exceptions près, d'une attitude désinvolte vis-à-vis de la fréquentation des églises, sans pour autant s'ancrer dans une forme de scepticisme envers le fait de croire. Les Baruya ne se considèrent jamais dans l'obligation de se rendre au culte, et ne vivent pas avec le «tourment moral »(Robbins, 2004a) généré ailleurs par une contradiction entre le système de valeurs existants et les nouvelles règles de l'Église. On pourrait alors voir dans les Dix Lois citées cidessus une forme de justification de l'absence de nécessité d'aller à l'église, puisque l'on en connaît déjà les principes. Ceux qui se disent "non chrétiens " me confient effectivement " savoir déjà de quoi on y parle", et revendiquent ainsi le caractère indigène de règles exogènes ; ils ne se sentent jamais exclus de cette communauté de pensée, ou de la modernité que l'on associe localement à l'arrivée du christianisme dans la vallée.

À l'église, on lit des extraits de la Bible en tok pisin (traduits en langue yipma), on chante et joue parfois de la musique, mais surtout les $\mathrm{Ba}$ ruya utilisent le moment de l'office comme plateforme pour parler d'autre chose, de problèmes situés en-dehors du domaine religieux, mais susceptibles d'intéresser la communauté, exac- tement comme lorsqu'ils se rassemblent sur la piste d'atterrissage le dimanche après-midi pour discuter de choses et d'autres. Autrement dit, le culte fournit un moment privilégié de rassemblement permettant d'aborder des problèmes et des questions qui lui sont extérieurs et que la religion chrétienne n'a pas pour but de résoudre, mais qui occupent les esprits. C'est la dimension religieuse qui tend à s'effacer du service, au lieu de gagner d'autres aspects de la vie sociale. Au service des luthériens célébrant la Pentecôte en 2013, aucune mention ne fut faite du Saint-Esprit ou de la Trinité, mais le pasteur parla surtout de problèmes d'argent de tout un chacun dans un endroit aussi isolé, et de l'absence d'action du gouvernement. Avant et pendant l'office, le principal sujet de discussion concerna les doléances des habitants de la vallée ${ }^{12}$. Un ancien pasteur vint au pupitre lire l'épître de Saint Paul aux Romains $13: 1-7$, ce qui mena à une discussion sur l'autorité et sur le rôle très limité du gouvernement. Précisément, ce passage de la Bible résume les Dix Commandements et en souligne l'importance :

12. Les problèmes se résument à l'absence de route menant en ville; une telle route apparaît pour les Baruya comme la solution à tous leurs problèmes, car il leur serait possible d'acheter des denrées à moindre coût, et d'aller y vendre leur café vert en négociant le prix à la hausse. 


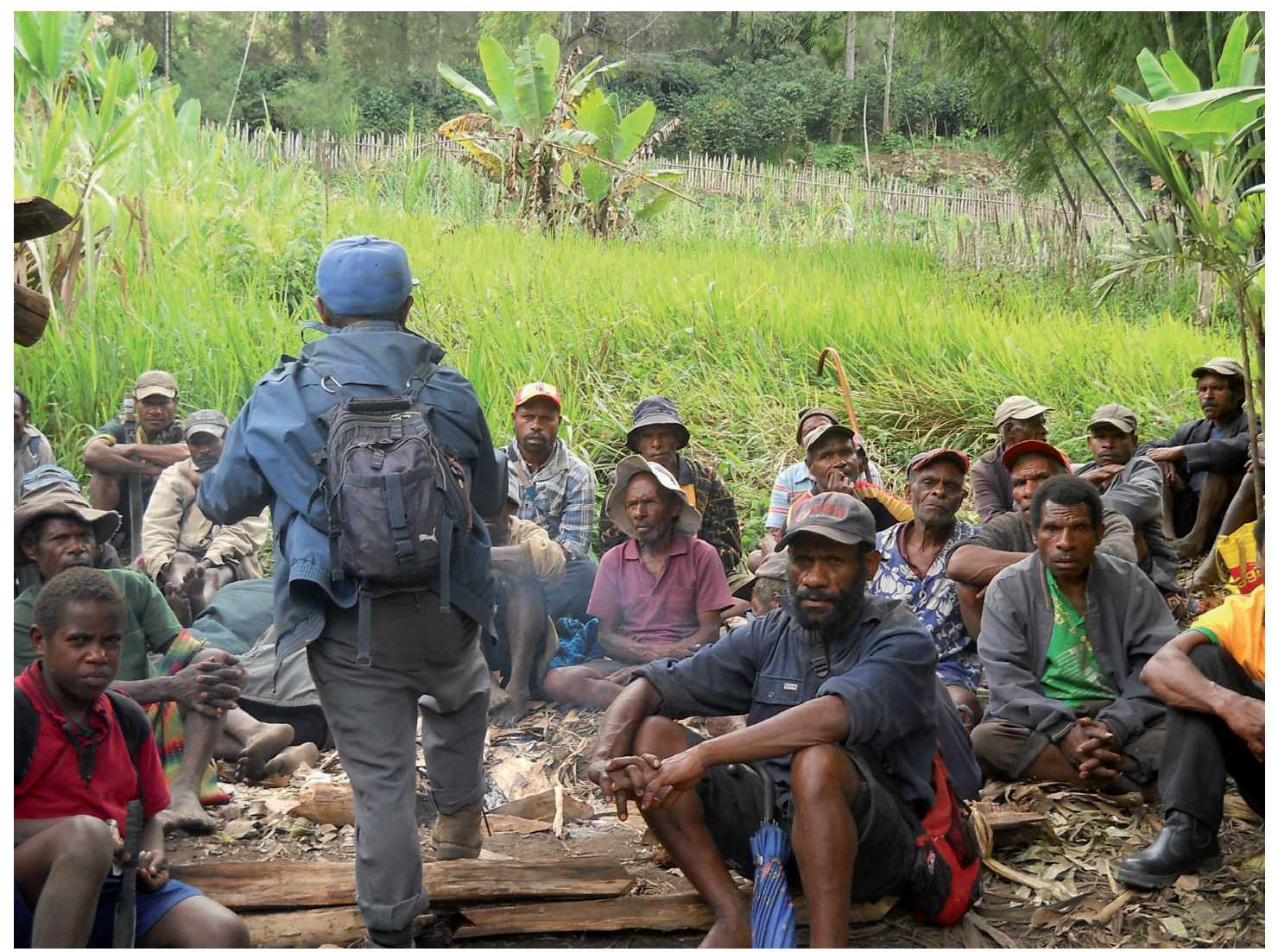

Рното 3. - Durant un enterrement, les hommes se rassemblent pour parler de politique, village de Yanyi, juin 2013 (@) Anne-Sylvie Malbrancke)

« Ne devez rien à personne, si ce n'est de vous aimer les uns les autres ; car celui qui aime les autres a accompli la loi ; En effet, les commandements : Tu ne commettras point d'adultère, tu ne tueras point, tu ne déroberas point, tu ne convoiteras point, et ceux qu'il peut encore y avoir, se résument dans cette parole: $\mathrm{Tu}$ aimeras ton prochain comme toi-même " (version Louis Segond).

Il est remarquable que ce soit cet extrait qui ait été lu devant l'assemblée des fidèles durant ce service de Pentecôte: lors de ce service, on ne parla que d'un code moral décroché du sacré, en un jour où précisément les chrétiens célèbrent d'ordinaire l'Esprit Saint venu se poser sur la tête des fidèles. Le pasteur parla aussi des problèmes récurrents de grossesses hors des liens du mariage, et relia ceci à la disparition de traditions permettant d'assurer un " comportement décent " dans la vallée. À aucun moment l'Église n'est apparue dans son sermon comme une entité ayant le rôle de régler ces problèmes.
En cela, le contraste est net avec les Urapmin du Sepik occidental, où le christianisme charismatique a été élevé au rang de valeur religieuse centrale (Robbins, 2004a). Tandis que ces derniers diabolisent les croyances ancestrales, chez les Baruya ce sont des chrétiens qui sont l'objet de moqueries ${ }^{13}$, accusés par leurs contemporains de dépravation du corps comme de l'esprit ( $p a-$ muk lain, litt. " ceux qui sont dépravés»). Effectivement, les Baruya interrogés qui ne s'identifient pas (ou plus) comme chrétiens accusent les pratiquants d'aller à l'église uniquement pour séduire l'autre sexe, et me disent avec assurance qu'ils sont nombreux à quitter le service en plein milieu pour s'adonner aux plaisirs de la chair. Ils emploient aussi volontiers l'insulte de pamuk lain parce qu'ils comparent les pratiquants allant d'une confession à l'autre aux individus qui ont des rapports sexuels avec différents partenaires. Car chez les Baruya, il est rare de fréquenter la même Église tout au long de sa vie ${ }^{14}$. Cela ne remet pas nécessairement en cause leur croyance

13.À cet égard, le concept d'humiliation (Robbins, 2005a, 2005b) ne s'applique pas à la culture traditionnelle baruya, qui est glorifiée de manière presque unanime, par les chrétiens comme par les autres. Elle s'applique, en revanche, à certaines pratiques chrétiennes, que les non pratiquants voient facilement d'un oil critique et narquois.

14.Cependant les femmes ne sont pas obligées de quitter leur confession pour suivre celle de leur mari, comme ailleurs en Papouasie Nouvelle-Guinée (par exemple chez les Gebusi de la Western Province, d'après mes propres observations sur le terrain en mai-juin 2016 ; chez eux il s'agit de l'unique forme de " nomadisme ecclésial »). 


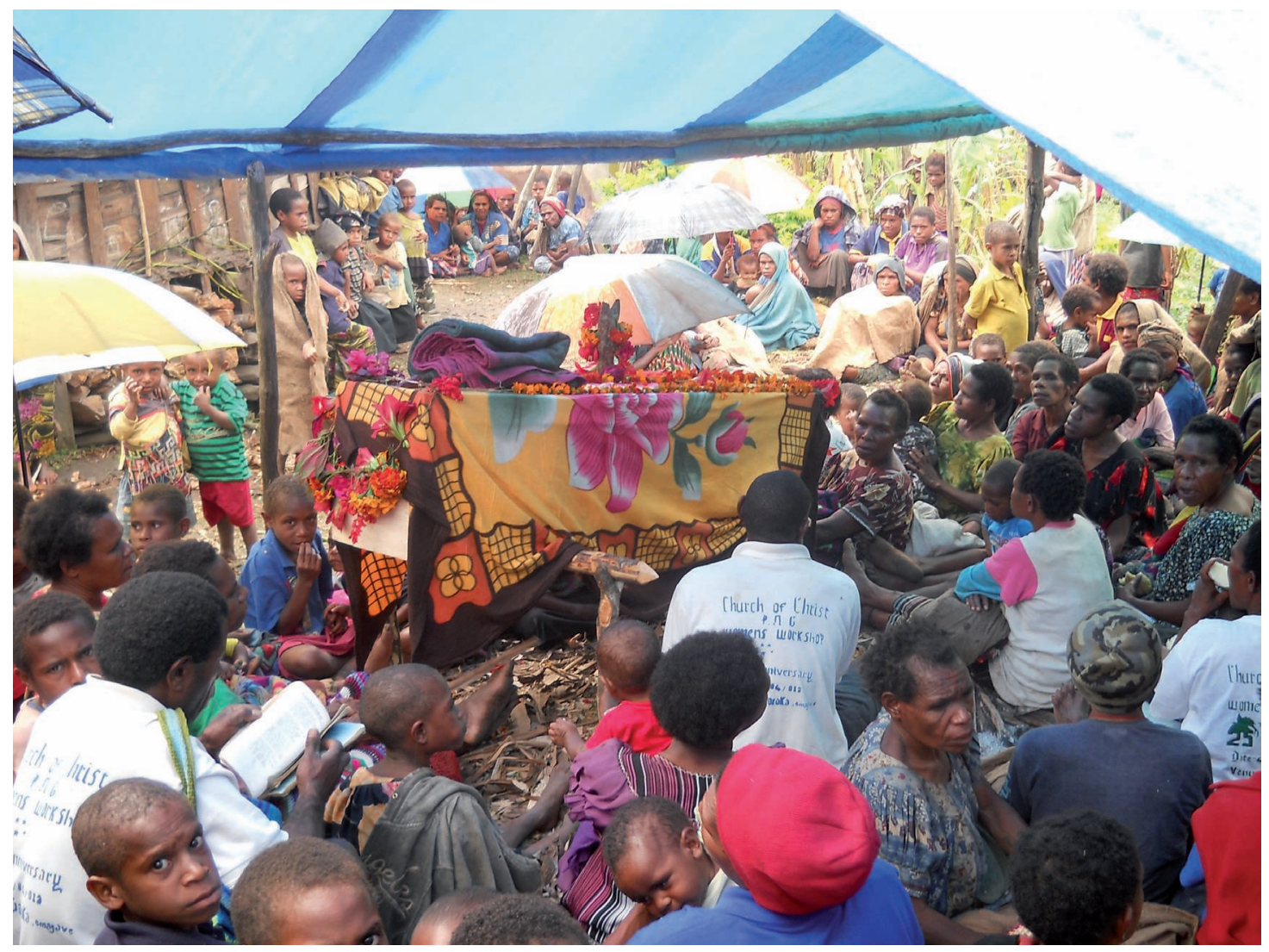

Рното 4. - L'enterrement d'un homme âgé chrétien (Church of Christ) à Yanyi, Wonenara, juin 2013 (C) Anne-Sylvie Malbrancke)

dans le christianisme, mais cela souligne une certaine flexibilité dans l'approche des diverses confessions présentes, dont les différences théologiques ne sont pas marquées. D'ailleurs l'expression tok pisin employée pour déclarer que l'on est chrétien se traduit littéralement par «aller à l'église " (go long lotu), ce qui déforme en dernière instance la notion d'être chrétien. Mais peut-être est-ce là une assez juste réfraction de la relation que les individus entretiennent avec les Églises, tout au moins pour les Baruya : il s'agit moins de croire en des notions chrétiennes que de se rassembler le dimanche et d'agir en accord avec des préceptes que l'on identifie de toute façon aux valeurs morales indigènes.

Pour qualifier ce changement régulier de confession, Pascale Bonnemère parle de "nomadisme ecclésial "(2013 : 229). Parmi mes interlocuteurs adultes déclarant être ou avoir été pratiquants, les trois quarts font état d'un ou de plusieurs changements d'Église(s) au cours de leur vie - certains ayant été baptisés trois fois. C'est toujours une forme de pragmatisme qui est à l'origine du changement d'Eglise et de la conversion : les individus racontent qu'ils souffraient d'une maladie ou qu'ils perdaient beaucoup d'enfants, ou que leur vie ne les satisfaisait pas complètement, et qu'ils étaient à la recherche d'une solution. Par comparaison, Meyer (1998: 320) montre qu'au Ghana la conversion à une Église pentecôtiste est seulement en partie inspirée par le besoin de résoudre des problèmes en lien avec la santé et la prospérité ; in fine cette conversion est motivée par l'idée de la salvation individuelle, valeur centrale dans la culture chrétienne, mais très peu mise en avant par les $\mathrm{Ba}$ ruya. Le pragmatisme des Baruya est une forme de gestion de leur propre souffrance et de sublimation des carences ou des soucis constatés dans leur vie quotidienne, en premier lieu la maladie et la forte mortalité infantile. Ce constat ne nie pas la réalité de la conversion. Robbins souligne que certains anthropologues, eux-mêmes issus de pays chrétiens, se montrent sceptiques dès lors que la conversion est motivée par des raisons pragmatiques (2007a : 12). Or il n'est pas question ici de douter du fait que certains individus s'identifient comme chrétiens, mais il convient de noter et d'analyser ce que les Baruya euxmêmes retiennent de leur adhésion à un système religieux. Les considérations pragmatiques à la source du mouvement de conversion mènent à une grande flexibilité dans le rapport aux différentes confessions présentes, au baptême, à la parole divine, dans la mesure où le contenu théologique n'est pas à la source de la décision 
de conversion. Les Baruya ne font pas porter l'accent sur une rupture spirituelle, sur une forme d'introspection ou de transformation de la morale, offrant en cela un contraste frappant avec d'autres populations converties de Mélanésie ou encore d'Afrique (Robbins, 2004a ; Meyer 1999). À Wonenara, la conversion se situe sur un terrain d'où Dieu est remarquablement absent.

Peut-on pour autant conclure que les Baruya sont "moins" chrétiens que les Urapmin par exemple $?^{15}$ Estimer qu'une population est " plus » chrétienne qu'une autre impliquerait de voir dans le christianisme un ensemble immuable de règles à suivre, de croyances et de pratiques à adopter. Cette position n'est pas soutenue dans cet article, et ne ferait aucune place théorique à la variabilité même de la christianisation, portée par des missionnaires (locaux ou occidentaux selon les endroits) dont les positions et volontés d'adaptation au contexte local varient dans l'espace et dans le temps ${ }^{16}$. La volonté catholique d'inculturation est un exemple à cet égard éclairant : l'objectif avoué des missionnaires était alors de remodeler la culture évangélisée tout en adaptant la parole sacrée au contexte local. Céline Réjasse (2011) présente ainsi l'exemple d'un rituel "inculturé ", inventé par un prêtre italien dans une région de Madagascar particulièrement réfractaire au christianisme. L'auteure explique :

«L'inculturation, c'est avant tout la nécessité et la possibilité d'extraire l'Évangile de son carcan culturel d'origine, d'en faire un "objet" a-culturel et a-temporel, greffable sur n'importe quelle autre culture." (2011: 74, italiques dans l'original)

Malgré des convergences incontestables entre différentes confessions (voir aussi Knauft 2002), et un ensemble de dogmes canoniques ${ }^{17}$, le christianisme n'est pas un ensemble prédéterminé d'éléments qu'il faudrait retrouver intacts dans la culture christianisée pour parler de conversion. Notre propre catégorie de christianisme n'englobe pas les mêmes réalités que pour des populations christianisées : c'est à cette variabilité même du contenu de la catégorie et de son usage analytique qu'il faut prêter attention à un niveau ethnographique puis théorique. Il est admis et démontré ethnographiquement que chaque culture intègre divers éléments de la religion introduite de manière variable. L'idée d'une " culture chrétienne » doit-elle donc être éloignée tout à fait et ne peut-on rien dire du christianisme des Baruya par rapport à celui des Urapmin ? C'est là qu'une approche inspirée par le modèle développé par Robbins (voir notamment 2015) semble riche analytiquement : c'est en observant la relation entre les valeurs structurantes de la société étudiée que l'on peut espérer saisir l'impact fait par le christianisme et en déterminer l'étendue (plutôt qu'en décomptant la présence de telle ou telle pratique, telle ou telle croyance). Il ne s'agit pas tant de déterminer si ce modèle est confirmé ou infirmé par l'exemple baruya, mais de montrer le continuum qui existe entre différentes réalités ethnographiques et d'ouvrir la réflexion au concept de variation, en interrogeant les limites du modèle pour se demander à partir de quel point on peut estimer qu'il y a rupture et changement radical. Je vais m'efforcer dans la suite de cet article de démontrer que les modalités d'adoption du christianisme par les Baruya réduisent cette religion à un ensemble de valeurs morales identifiées aux valeurs locales et en limitent donc l'impact dans le changement socioculturel par ailleurs à l'œuvre chez les Baruya ${ }^{18}$.

\section{D'une loi à l'autre : séculariser la religion}

Effectivement, le nomadisme ecclésial des Baruya se comprend d'autant mieux à la lumière d'un discours holistique qui assimile les Dix Commandements de la Bible aux "Lois des ancêtres ", que l'on appelle aujourd'hui les "Dix Lois des Baruya " ("Tenpela Lo bilong ol Baruya» en tok pisin; l'expression n'est jamais employée en langue vernaculaire $\left.{ }^{19}\right)$. Un tel rapprochement était en germes dans les discours de certains pasteurs: Bonnemère rapporte que les EBC ont depuis longtemps cherché à établir des ponts entre récits bibliques, préceptes chrétiens

15.Je remercie grandement un relecteur/une relectrice anonyme pour avoir soulevé cette question, dont il est établi qu'elle est épineuse voire insoluble, mais qui ouvre malgré tout de riches perspectives de réflexion.

16. Comme le souligne un relecteur/une relectrice anonyme, il faut dissocier les positions dogmatiques des différentes confessions (elles-mêmes variables), des aménagements pragmatiques qu'elles autorisent, plus ou moins à la marge, ainsi que les points de rupture (ce qui est acceptable chez les Baruya ne le sera pas nécessairement ailleurs pour une même confession).

17.Comme le rappelle très justement un(e) relecteur(-trice) anonyme.

18. Il est vrai que parler de religion au singulier peut être problématique. Cependant, chez les Baruya comme dans d'autres régions du pays, les différentes confessions au même endroit semblent converger vers un contenu très similaire. Comme le montre Bruce Knauft pour les Gebusi de la Western Province (2002 : chap.6), se rendre chez les Adventistes, Catholiques ou chez les évangéliques (ECPNG) implique avant tout l'adoption d'un certain mode de vie (vêtements, alphabétisme, ponctualité). Les différentes confessions présentes convergent toutes vers une forme de fondamentalisme qui gomme les distinctions entre les cultes; chez les Baruya, ce phénomène se double d'une réduction des dogmes à un ensemble de valeurs qui ne se situent pas sur un plan théologique.

19. La totalité des 189 individus interrogés lors d'entretiens semi-dirigés conduits entre mai 2013 et avril 2014 connait et emploie cette expression, toujours en tok pisin. 
et mode de vie traditionnel, afin que les premiers ne soient pas tout à fait déconnectés du second (2013 : 248-249). Ces parallèles tracés entre deux logiques au fonctionnement similaire (des règles à respecter) mais aux contenus divergents (la teneur de ces règles) ont favorisé l'émergence d'un discours qui assimile tout à fait les deux ensembles de Lois - d'autant mieux que le contenu théologique chrétien est peu internalisé par les Baruya (Bonnemère, 2013). On peut ainsi, par exemple, défendre les initiations masculines et la fréquentation de l'église, qui "ne sont pas ennemies" (dixit Gesom, ancien pasteur luthérien, formé à la Bible School de Lae). L'incompatibilité de croyance spirituelle est rarement soulignée, mais elle existe: j'ai documenté deux cas seulement de familles ayant choisi de ne pas initier leurs filles en raison de leur adhésion au christianisme, voyant dans l'initiation une contradiction avec le culte luthérien auquel elles se rendaient toutes les semaines. L'antagonisme n'était cependant pas suffisant pour empêcher l'initiation de leurs fils qui n'auraient pas grandi "comme des hommes" sans ce rituel ${ }^{20}$. Ces accommodements nuancent l'idée que le christianisme aurait redéfini profondément les "valeurs » baruya : localement, on me dit qu'elles sont maintenues alors même que certaines pratiques et savoirs anciens disparaissent ou se recomposent - peut-être moins sous l'action du christianisme que d'autres agents de la modernité.

Yaruemmaye, qui cumule une fonction liée aux initiations masculines et un certificat de la mission adventiste, a accepté un jour de détailler les «Dix Lois Baruya ». Comme je l'ai annoncé en introduction, malgré la récurrence de cette expression dans les discours, il me fut difficile de trouver quelqu'un pour les énumérer dans le détail. Gillion, un homme de 45 ans, m'avait dit qu'elles étaient « secrètes " et qu'il ne pouvait pas les répéter à haute voix - il les traitait, en cela, comme le sont les règles des ancêtres transmises dans le cadre très ritualisé des initiations. Yaruemmaye, en revanche, se prêta volontiers à l'exercice. Il détailla en fait les onze lois citées supra (p. 228), le surnombre venant probablement du fait qu'il s'agit moins d'une liste établie et connue par cour que d'idées plus générales sur les valeurs qui font sens dans l'horizon moral baruya ${ }^{21}$. L'analyse des « commandements » listés en introduction permet d'en dégager certaines caractéristiques importantes.

Alors que la structure des Commandements bibliques est reprise, les contenus sont adaptés à une réalité locale. Cette liste reformule ainsi des lois provenant des ancêtres, réaffirmant, dans une poétique différente, certains des préceptes transmis lors des initiations masculines (Godelier, 1982 : 66). Dans la cosmologie baruya, le Soleil est le "père surnaturel " (Godelier, 1982 : 91) de tous les humains, une puissance qui fabrique les yeux, le nez, la bouche, les doigts et les orteils des foetus et qui transmet sa force aux hommes durant les initiations (Godelier, 1982: 72) et non une instance régulatrice surveillant les actions de chacun d'en haut. On voit là une inflexion de la logique traditionnelle vers une dynamique proprement chrétienne: une puissance supérieure observe tout un chacun, et punit en juge suprême. Ainsi mobilise-t-on, dans un cadre que l'on associe à la modernité, la cosmologie indigène ancestrale pour rendre compréhensibles les nouvelles normes, faisant dans un même mouvement une opération de traduction et l'affirmation d'une continuité avec les représentations ancestrales.

Inversement à cette christianisation de l'Univers baruya, on peut également voir la rhétorique de ces Dix Lois plier la religion chrétienne aux règles de vie, au symbolisme ${ }^{22}$ et à l'échelle des valeurs baruya, subordonnant les valeurs chrétiennes à des valeurs locales " englobantes " (Robbins, 2010a). Les interdits les plus graves - et les plus récurrents - portent sur la question du vol. Ici, la déclinaison des objets cités faisant l'objet d'un interdit (femme, terre, bétel, pandanus) suit l'ordre de leur importance sociale. Ces possessions sont fondamentales pour la subsistance comme pour la sociabilité (la noix d'arec est par excellence l'objet de l'échange). Elles doivent être particulièrement respectées pour assurer la pérennité de l'ordre social. Pourtant, et comme le montre l'histoire de la femme enceinte ayant volé des barres de sel racontée plus bas, cette liste ne prétend pas à l'exhaustivité. Le but de cette emphase sur le vol, selon moi, est de souligner la gravité d'un crime, quel qu'en soit l'objet, non d'en limiter le champ ${ }^{23}$. À cette répétition du vol

20.Cette approche différentielle des initiations masculines et féminines fait écho à la moindre importance des secondes par rapport aux premières (voir notamment Godelier, 1982 : 84).

21. On pourrait se demander si ces lois précises n’ont pas été inventées sur le coup, pour me montrer qu’elles existent alors que l'expression désigne en fait un ensemble relativement indéterminé de valeurs fondamentales, comme le souligne la citation rapportée en introduction de cet article.

22. Le pandanus est valorisé pour les graines qu'il produit, mais également parce qu'il représente traditionnellement l'" arbre-mâle par excellence ", et jouait un rôle important lors de la cérémonie d'initiation d'un jeune garçon dont la fiancée venait d'avoir ses premières règles (Godelier, 1982: 69).

23. On m'encouragea ainsi un jour à blâmer en public un jeune homme qui avait volé le stylo que j'avais offert à sa sœur cadette. Mes interlocuteurs trouvaient qu'il méritait de prendre des coups ; il est vrai que cet objet était porteur d'un certain prestige puisqu'il venait d'un autre pays et était convoité par beaucoup. Cependant le crime est tout aussi marqué pour le vol d'épis de maïs dans un jardin (les jeunes gens reconnus coupables avaient subi l'opprobre général), et l'on excuse souvent le comportement violent d'une victime de vol cherchant à se faire justice elle-même. Ainsi un chamane ayant un jour perdu une pelle, convaincu qu'elle lui avait été volée, avait menacé tout le village de représailles terribles, et se sentait investi du droit de 
comme crime toujours condamnable s'oppose l'ambivalence du meurtre, qui n'est pas toujours interdit dans l'absolu. Cette relativité explique un paradoxe : tuer un homme est interdit (troisième Loi) alors que c'est un devoir, pour un Baruya, de tuer un ennemi (Godelier, 1982 : 66), comme le rappelle la neuvième Loi. Comme pour le vol, les Lois classent donc la gravité des meurtres en une série intégrant les enfants d'autres hommes, la famille et les hôtes (des commensaux) avec toutefois ici, comme net contrepoint, l'injonction de tuer les ennemis. Car pour les Baruya, une distinction demeure entre les hommes baruya et les autres, qui ne sont pas tout à fait des hommes. La doctrine chrétienne selon laquelle tous les hommes sont égaux n'est pas concevable. Notons par ailleurs que l'idiome du sang pour parler de la famille est un fait assez récent, informé par une transformation progressive du symbolisme des substances corporelles : le sperme était autrefois vu comme la substance vitale par excellence (Godelier, 1982: 91, 99, 108), transmise en ligne agnatique et définissant l'appartenance à un même lignage (Godelier, 2009: 48). Je montre ailleurs le glissement de l'idiome du sperme vers l'idiome du sang pour désigner le partage d'une identité lignagère (Malbrancke, 2016a: 146), ainsi que la dévalorisation croissante du symbolisme du sperme, associé aujourd'hui par les plus jeunes aux infections sexuellement transmissibles (Malbrancke, 2016a : 151).

Ces lois ne sont pas un code strict et connu de tous que l'on invoquerait dans des cas de conflits ou lors des tribunaux de village ("village courts») même si "l'esprit de la loi coutumière " est bien connu d'Elijah Nokaï, figure locale d'une autorité issue du gouvernement. Il m'expliquait, sans faire nommément référence aux "Dix Lois", qu'il devait composer avec la loi papoue issue de la Constitution et avec la loi coutumière dans son arbitrage des conflits autour de la terre, des vols, des adultères, des violences conjugales. Ces affaires, les plus courantes dans la vallée, se règlent par une compensation monétaire parfois remplacée par de la viande de porc, pour recréer par la commensalité les relations de bonne entente. $\mathrm{Ce}$ règlement somme toute rapide de l'offense évacue tout concept de "transcendance " de la punition. Mais alors que les lois papoues (en matière de violence conjugale par exemple) ne portent pas à conséquence (ou stigmate) dans la vie quotidienne, les infractions aux règles que listent les Dix Lois baruya entrainent un fort stigmate social, ce qui montre l'importance de ces valeurs pour les Baruya ${ }^{24}$. Bien que le vol, par exemple, soit soumis à compensation, comme d'autres offenses, il est très difficile de se faire pardonner un tel crime. En février 2014, une femme mariée et enceinte fut accusée d'avoir volé des barres de sel ${ }^{25}$ à une voisine; l'affaire était grave, mais l'accusée fut menée dans une petite hutte pour accoucher dans les jours qui suivirent. Alors que l'on parlait encore de son vol, elle commença à se plaindre de douleurs violentes qui durèrent plusieurs jours après l'accouchement. Personne ne put trouver la cause de ses maux, et les médicaments que je lui fournissais ne semblaient pas faire effet. On l'avait même menée en civière jusque dans le bas de la vallée, pour être examinée à la clinique de brousse (haus sik), sans succès. D'aucuns dirent qu'il s'agissait d'une manifestation de sa culpabilité, d'autres parlèrent de "comédie " visant à faire oublier son méfait. Mais son crime resta dans tous les esprits, et occupa bien des discussions dans les semaines qui suivirent, période pendant laquelle personne ne lui rendit visite et où elle resta dans sa maison. Si le vol est toujours clairement inacceptable, le meurtre à l'inverse est parfois prescrit, surtout aujourd'hui où la menace de guerre avec les Ipmani rejaillit après plusieurs années de paix $^{26}$ : autrement dit, dans cette for-

brûler la maison du coupable, même si sa famille devait périr dans les flammes. En d'autres termes, cette liste d'interdits portant sur le vol ne viserait pas à circonscrire le champ du commandement, mais au contraire à en montrer l'étendue, potentiellement infinie. L'effet rhétorique de la répétition, qui pourrait nous sembler en fait limiter l'application du commandement (hors de ces possessions, le vol serait toléré) est plutôt selon moi dans le discours baruya une façon de renforcer l'interdit. Il est bon de souligner ici que Yaruemmaye ne s'arrêta pas à Dix Lois, mais continua, comme si l'énumération pouvait en fait être infinie.

24.La question du viol présente des nuances intéressantes : j’ai constaté lors d'un tribunal de village réuni en février 2014 suite à une agression sexuelle que l'offense était nettement aggravée par les liens de parenté qui reliaient les deux jeunes gens. Dans sa façon d'exposer le crime, et pour justifier la forte compensation demandée, le magistrat fit clairement figurer cette valeur centrale, ce facteur aggravant qu'était la relation de cousins croisés. Or cette forme de " consanguinité ", a connu une transformation très nette : aujourd'hui deux cousins croisés sont dits trop " proches " pour se marier, alors qu'il s'agissait d'une alliance autrefois idéale (voir Godelier, 1982 : 45 et Malbrancke, 2016a : 138, 144). En ce sens, les valeurs baruya informent aussi largement la sphère du mariage, mais celle-ci est laissée de côté par Yaruemmaye dans son énumération des lois; dans la suite de l'entretien, il m'expliqua que les règles relatives au mariage étaient transmises, notamment par lui-même, lors des initiations masculines. On disait alors aux jeunes garçons qu'il leur était interdit de quitter leur épouse, et que s'ils convoitaient la femme d'un autre ils risquaient la mort. On leur disait aussi de n'avoir que 3 ou 4 enfants. On le voit, les valeurs des Baruya sont loin d'être limitées à l'énumération qui m’en a été faite, mais elles subsistent après l'introduction du christianisme, et sont réaffirmées par son truchement, tout en dépassant ce cadre de transmission.

25.Les barres de sel de potassium sont traditionnellement utilisées comme monnaie dans les échanges inter-tribaux (Godelier, 1969). Je postule qu'elles ne faisaient pas partie des objets listés par Yaruemmaye dans la liste des interdits spécifiques relatifs au vol précisément parce que cette liste ne se voulait pas exhaustive.

26. Menace réactivée précisément par le vol de plants de café par des Ipmani sur des terres baruya (mais dont la propriété est contestée depuis la fin de la guerre qui les opposa dans les années 1980). 


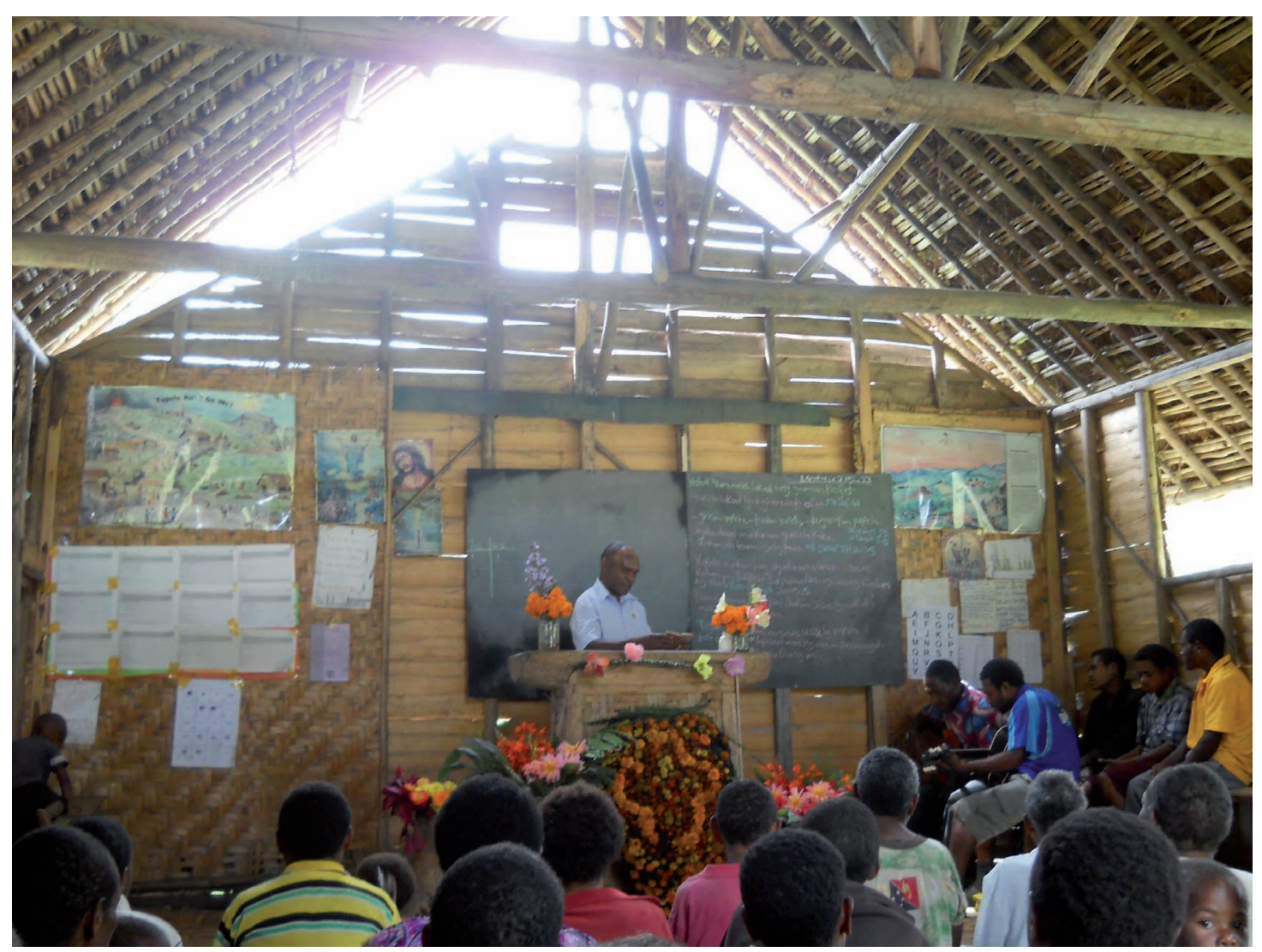

Рното 5. - Un prêche dominical délivré par un Baruya laïc polygyne (Église EBC, mai 2013) (@) Anne-Sylvie Malbrancke)

mulation de leur modernité, les Baruya réitèrent et renforcent certaines valeurs traditionnelles ${ }^{27}$.

Ce sont donc des aspects non religieux de la vie sociale qui sont formulés dans un idiome religieux: c'est ce qui relève des valeurs civiques de la vie sociale baruya qui est formalisé en commandements. Cela mène l'analyse à une redéfinition de ce que pourrait être la religion chrétienne dans cette perspective, puisque seuls certains éléments décrochés du sacré en sont conservés. Le constat revient, globalement, à une réappropriation de la religion, qui en conserve principalement la codification des pratiques et la gestion des relations sociales, c'est-à-dire son versant séculier. La référence à la Bible sert ainsi d'idiome pour exprimer des valeurs dont cette dernière n'a pas le monopole et renforce ainsi la revendication indigène d'un système de valeurs perçu comme moderne. Chez les Baruya, ce n'est pas l'idéologie chrétienne qui est première, et qui redéfinirait les valeurs locales : dans cette optique, l'acceptation de la polygynie par les Églises présentes à Wonenara m’avait mise dès le début de mon terrain sur la piste d'une adaptation des valeurs chrétiennes aux mœurs locales plutôt que l'inverse $^{28}$ (voir aussi Bonnemère, 2013 : 252). La question qui se pose alors est moins celle de la "véracité " de la conversion des Baruya que celle de l'impact du christianisme sur la culture baruya et de son rôle effectif dans le changement socioculturel.

\section{Adopter et adapter le christianisme dans un contexte changeant}

Chez les Baruya, l'intégration de la rhétorique et de références d'origine chrétienne, parfois même une conversion fervente, ne font pas l'objet de

27.Le vol est d'ailleurs l'image qui informe la perception du comportement des «tukina meri», qui couchent avec des hommes en échange d'une somme d'argent : hommes et femmes parlent de ces dernières comme de "voleuses " ("stilmeri») car elles volent l'argent d'un couple, et sont vues par les femmes comme capables de "voler " un mari (en le détournant de son épouse). En ce sens, les règles détaillées ci-dessus ont une inflexion chrétienne dans leur formulation (ne pas " convoiter la femme d'autrui »), mais l'interdit vaut largement pour les deux sexes, ce que reflète là aussi le stigmate tenace qui marquera le responsable d'un tel «vol».

28.Par contraste, la situation décrite par Meyer au Ghana (1998 : 320) fait état d'une interdiction stricte de la pratique de la polygynie pour tout adhérent d'une Église pentecôtiste. 
résistance(s), même de la part des non-croyants. Ladoption de la rhétorique chrétienne relie les convertis comme les autres autour d'un noyau partagé de valeurs, dont la force et la légitimité sont réactivées sur deux fronts, celui de la tradition ancestrale et celui d'une modernité venue de l'extérieur. C'est par cette rhétorique que passe la revendication de conversion, d'autant plus convaincante et positive aux yeux de mes interlocuteurs qu'elle ne pose pas le moindre problème de compatibilité avec une tradition par ailleurs unanimement glorifiée. Ceux qui n'adhèrent pas au christianisme n'en acceptent pas moins l'existence de Dieu et ne rejettent pas, en principe, les valeurs exogènes. Ils n'y trouvent pas d'intérêt, ces valeurs faisant déjà partie de leur quotidien.

Avec le concept de "structure des conjonctures ", Sahlins fournit le moyen de penser l'inséparabilité entre continuité et différence (1981: 68). Lors de la rencontre entre la structure et l'événement singulier (en l'occurrence l'irruption du christianisme), les codes des deux systèmes ne coïncident pas nécessairement, mais une forme de compréhension mutuelle a lieu. L'ensemble de rapports historiques (la conjoncture) reproduit les catégories culturelles (la structure) et leur donne de nouvelles valeurs : le christianisme est vu et intégré comme un système dont certaines valeurs (reformulées et adaptées) auraient existé longtemps avant l'arrivée des missionnaires dans la vallée. Ainsi l'adoption de la poétique chrétienne sert de support à son intégration et à sa compréhension dans un contexte qui a été bouleversé, mais où, malgré les changements économiques, politiques et sociaux, la hiérarchie des valeurs subsiste. Certes ses principes sont reformulés et plus éloignés qu'autrefois d'une forme de sacré relié à une cosmologie ancestrale, mais dans le même temps, le christianisme n'est adopté que par fragments, et en sont conservés les aspects qui peuvent faire sens au sein de la société baruya actuelle. Ce sont les analogies structurelles qui sont privilégiées et mobilisées suite à la reformulation des lois ancestrales sous forme de "Dix Commandements ", loin de tout mouvement de rupture et de rejet. Comme Barker le décrit chez les Maisin de Papouasie Nouvelle-Guinée (2012a), christianisme et système religieux indigène peuvent parfois être intelligibles entre eux (sous certaines conditions, variables dans l'espace et le temps), sans que l'un soit la directe continuation de l'autre : il reprend Sahlins pour rendre compte d'une telle situation, parlant d'une "structure des conjonctures" où le contexte local permet une transposition structurelle de l'imaginaire exogène au sein de schémas endogènes de valeurs. Cette transposition ne préjuge pas de l'existence, par ailleurs, de changements sociaux : comme Barker le dit, la conversion a entraîné chez les Maisin des changements parfois radicaux, qui ont nécessité l'adoption consciente de la tradition et de la modernité dans le même temps (Barker, 2012a : 48). Ce qu'il cherche à penser, ce n'est pas la continuité culturelle ou la résistance, mais ce qui émerge comme produit de la rencontre entre deux systèmes moraux et spirituels (Barker, 2012a : 54).

Malgré l'indéniable force de rupture qui accompagne l'arrivée du christianisme et que l'anthropologie récente du christianisme a volontiers soulignée (Meyer, 1998 ; Engelke, 2004 ; Robbins, 2004a ; Haynes, 2014), des études s'attachent aussi à démontrer l'impact d'autres phénomènes, comme par exemple les réseaux économiques et politiques transnationaux (Hann, 2007 ; Comaroff, 2010), jugés plus saillants dans la fabrique de la différence et de la rupture que le christianisme. Dans la mesure où les Baruya retiennent du christianisme une rhétorique qui leur permet de formuler leurs propres valeurs dans un cadre perçu comme moderne, ce n'est pas l'introduction de cette religion qui serait à l'origine des changements sociaux observés chez eux. On pourrait, à la suite de Robbins (2004b), interroger la façon dont cette religion affecte plus largement les idées locales relatives au genre, à la politique, à l'économie, etc., moins par son contenu théologique que par sa structure institutionnelle. Chez les Baruya, les relations entre les sexes étaient autrefois marquées par une asymétrie très nette, reléguant les femmes à une position de soumission symbolique, matérielle, idéologique et politique. Effectivement, sans pouvoir de décision sur leur propre destin individuel, notamment marital (Godelier, 1982 : 222), elles étaient également exclues de la possession de la terre, comme de la connaissance des savoirs sacrés et reléguées à une place mineure dans les décisions relatives à l'intérêt général (Godelier, 1982 : 223). Leur place périphérique sur la scène publique était légitimée et renforcée par une idéologie essentialiste des fluides corporels : je l'ai dit plus haut, le sperme était la substance vitale par excellence, opposée en cela au sang menstruel, perçu comme essentiellement polluant et affaiblissant (Godelier, 1982 : 99). La "domination masculine" ainsi ancrée et justifiée en nature est largement en passe de s'effacer : on le constate d'emblée à la nette diminution de la ségrégation entre les sexes (à l'exception de la maison des hommes, de la rivière où l'on se lave et... de l'église). Les initiations masculines n'instaurent plus une distinction radicale entre les deux sexes, et même si elles maintiennent le processus par lequel on fait craindre les femmes aux jeunes initiés ${ }^{29}$, les

29. La muka, ou premier stade des initiations, a eu lieu en décembre 2013. À ce moment-là, on avait réuni les jeunes garçons de la vallée en âge d'être initiés dans la maison des hommes. On me dit qu'aucun précepte ne leur avait été transmis, mais qu'on leur avait déjà appris à craindre les femmes. Malgré la tenue de ces initiations, il semble que tout un pan de la culture ancestrale soit aujourd'hui ignoré des générations les plus jeunes: les mythes notamment leur sont inconnus, et 
relations changent progressivement du fait de la mixité des sexes, qui est maintenant la norme, sur les bancs de l'école comme parmi les groupes de gens jouant aux cartes. Avec les transformations idéelles relatives aux corps et à la reproduction ${ }^{30}$, c'est l'autorité masculine qui est sapée en son fondement (Godelier, 1982: 99).

Or quel rôle le christianisme a-t-il joué dans ces métamorphoses ? Bonnemère (2016) montre qu'à Wonenara, les reconfigurations des relations entre les sexes au sein d'une Église donnée dépendent de l'origine du pasteur célébrant l'office : là où des hommes baruya parlent aux fidèles assemblés, leurs conceptions culturelles relatives à la place dévolue aux femmes ont tendance à informer une vision conservatrice du rôle des femmes pendant l'office. À l'inverse, si le pasteur est extérieur à la tribu, les femmes trouvent au sein du culte plus d'espace pour être visibles et audibles, et voient dans la religion chrétienne un nouveau moyen d'expression et une place dans les rituels qu'elles n'avaient pas auparavant ${ }^{31}$. C'est dire que, tel qu'il est instauré, le christianisme n'est pas chez les Baruya une institution qui permet d'établir une rupture d'avec les normes culturelles traditionnelles. Au contraire, elle peut parfois les renforcer. La question est autant de trouver " the difference Christianity makes " (Cannell, 2006), que de trouver où il fait la différence, et où il ne la fait pas. Ce sont là des questions générales importantes à prendre en considération pour informer et raffiner de tels modèles de changement socioculturel. Car s'il est indéniable que les rapports entre les sexes chez les Baruya se sont transformés depuis leur premier contact avec les Blancs en 1951 (Malbrancke, 2016ab), le christianisme aurait eu un rôle limité dans ces métamorphoses, comme le conclut Bonnemère :

"The Baruya situation seems closer to the Huli one as no value associated with Christianity has as yet come to the fore. " (2016: 219)

\section{Conclusion}

En se convertissant au christianisme, les Baruya adhèrent à des valeurs qui sont en dernière instance les leurs. Leur identification à une doctrine chrétienne amputée de larges pans de sa théologie et de sa mystique ne leur demande pas une rupture ou un rejet de leurs valeurs traditionnelles; il ne s'agit pas pour eux d'un point de non-retour, mais d'un point d'articulation. Tout comme les Urapmin, les Baruya ont développé "their Christianity along the lines of their own choosing" (Robbins, 1997 : 37) ; ce faisant ils ont intégré ce christianisme à une vision endogène du monde, dont les valeurs principales n'ont pas été radicalement transformées par leur mouvement de conversion. Cette réflexion ne vise pas à conclure que chez les Baruya «le nouveau est similaire à l'ancien " (comme Robbins le reproche à beaucoup d'analyses sur la question, 2010b : 242; voir aussi Bialecki et al., 2008: 1144). Mais il convient d'adopter une approche nuancée, et de suivre ce que disent les Baruya eux-mêmes de leur rapport à la religion chrétienne. Comme le rappelle Robbins, l'ethnographe doit prendre en compte la perspective émique des phénomènes, et s'attarder sur les manières locales de comprendre le rapport au christianisme (2010b : 243). De même que l'on ne peut tout à fait ignorer ce que les Urapmin disent de leur changement dramatique, il faut souligner que ce sont les Baruya eux-mêmes qui affirment la proximité (voire l'identité) entre les deux ensembles de règles - qu'ils superposent tout à fait à ce que signifie croire en Dieu. On observe ainsi, à écouter ce que disent les Baruya de leur rapport au christianisme, que leurs valeurs fondamentales (dans une approche dumontienne) n'ont guère changé, même si la forme de leur expression est devenue chrétienne. Il ne s'agit donc pas de refuser ou d'interroger le fait que les locaux s'identifient comme chrétiens (ou non), mais plutôt d'articuler de telles déclarations avec nos propres catégories analytiques. Partant de prémisses essentialistes et réifiantes, il est analytiquement peu pertinent de se demander si telle population est "plus chrétienne " qu'une autre. On peut, en revanche, chercher à comprendre le rapport que les individus entretiennent entre un système de croyances indigène et un système exogène ainsi que la hiérarchie qu'ils instaurent entre les deux. Cette hiérarchie (le rapport des valeurs formant système) varie de manière significative d'une population christianisée à une autre. Une pensée de l'articulation entre identité, valeurs locales et nos propres concepts semble heuristiquement plus riche ici.

Non seulement il faut appréhender rupture et continuité à la fois dans une même société,

eux-mêmes me disent ne pas s'y intéresser car « c'est le temps de l'école maintenant... On veut comprendre le monde, par la science ». Ce témoignage me fut donné par Augustin, jeune homme d'une vingtaine d'années qui lui-même ne fréquentait pas l'école primaire locale faute de place et qui ne se rendait pas à l'église non plus, faute d'intérêt pour le culte.

30.Si le rôle des femmes dans la conception de la procréation était autrefois nié (Godelier, 1982 : 90, 213), il est aujourd'hui reconnu par de plus en plus de mes interlocuteurs, surtout les femmes.

31. On pourrait alors se demander si l'inscription du code moral local au sein du christianisme est avant tout une construction idéologique masculine, renforçant des codes traditionnels là où précisément ceux-ci risqueraient de changer (rappelons que le public assemblé à l'office est majoritairement féminin dans toutes les confessions et que tous ces discours sur les Dix Lois permettent aux hommes de se rapprocher d'une forme de modernité avec laquelle ils sont de facto moins associés). 
mais il faut pouvoir admettre qu'un même agent ait historiquement cherché à produire l'un ou l'autre, pour mieux s'implanter ou mieux rassembler les fidèles : ainsi les luthériens, aujourd'hui les plus tolérants de toutes les dénominations présentes dans la vallée ${ }^{32}$, ont autrefois attaqué frontalement les traditions locales, cherchant notamment à interdire les initiations masculines (Godelier, 1982 : 305). C'est alors qu'une distinction entre christianisation primaire et secondaire devient riche analytiquement, car l'arrivée de nouvelles confessions s'est produite dans un paysage déjà christianisé33, ce qui pourrait aussi rendre compte d'un discours local faisant état d'une familiarité avec le christianisme $e^{34}$ (Barker, 2012b). Dans leur évangélisation constante d'une vallée encore vue comme en partie païenne (Lemonnier, 2013 : 215), les stratégies des Églises passent autant par la fabrication de la ressemblance que par celle de la discontinuité. Ces différentes vagues et sédimentations de christianisation varieront d'un endroit à l'autre et informeront aussi la relation des locaux à la religion ainsi introduite. La variabilité de ce contexte historique plus large où s'inscrit la christianisation offre une autre résistance à l'idée d'un seul modèle qui pourrait permettre de comprendre partout les modalités de la conversion au christianisme.

Le concept de « structure des conjonctures » développé par Sahlins (1981) nous permet d'éclairer le fait chrétien chez les Baruya. Ceux-ci en font le moyen d'une inscription indigène dans la modernité, en remodelant et en réactivant des valeurs locales ancestrales, qui trouvent une nouvelle justification au sein d'un contexte modifié. La rhétorique chrétienne est employée et détournée comme instrument de renforcement et de légitimation de valeurs traditionnelles, tout en permettant de soutenir la revendication d'une modernité dont l'existence aurait ainsi précédé l'arrivée des Églises dans la vallée. Le discours qui soutient l'adhésion au christianisme permet de créer cette continuité entre deux systèmes de valeurs, tout en omettant l'essentiel de ce qui est propre au christianisme, qui reste largement méconnu dans son détail théologique et spirituel.

Il faudrait encore déterminer dans quelles conditions la conjoncture provoque parfois une
« réévaluation " des orientations locales au point de créer une rupture d'avec le passé, par une recomposition de la structure indigène elle-même. Pour Sahlins, ce point de non-retour est atteint lorsqu'on en vient à constater des phénomènes d'humiliation (1992: 24), comme fait culturel et comme état psychologique. Il reste toutefois à examiner comment et pourquoi les formes d'humiliations (marginales chez les Baruya et qui, à la rigueur, prennent pour cibles ceux qui se rendent au culte) peuvent être localement assimilées en termes de catégories indigènes tout en étant capables de créer un mouvement de rupture au point de « déloger " ces mêmes catégories (Robbins 2005a : 15). De telles perspectives de questionnement pourraient permettre de raffiner le modèle du changement socioculturel. À cet égard, une approche diachronique offre la possibilité d'appréhender plus finement la complexité d'une société sur le long terme et d'espérer ainsi saisir les façons variables dont le changement socioculturel opère dans diverses sphères de la vie sociale.

\section{BIBLIOGRAPHIE}

BARKER John, 2001. Afterword, Journal of Ritual Studies 25(2), pp. 105-108.

-, 2012a. The Enigma of Christian Conversion: Exchange and the Emergence of New Great Men among the Maisin of Papua New Guinea, in Laurent Dousset and Serge Tcherkézoff (eds), The Scope of Anthropology: Maurice Godelier's Work in Context, New York, Oxford, Berghahn Books pp. 46-66.

-, 2012b. Secondary Conversion and the Anthropology of Christianity in Melanesia, $\mathrm{Ar}$ chives de sciences sociales des religions 157, pp. 67-87 (http://assr.revues.org/23633, consulté le 08 août 2016).

Bialecki Jon, Naomi Haynes and Joel RobBINs, 2008. The Anthropology of Christianity, Religion Compass 2/6, pp. 1139-1158.

Bonnemere Pascale, 2013. À chacun sa Bible. Styles de prêche et rapport à Jésus dans la val-

32.Ils autorisent notamment la consommation de viande de porc et la mastication du bétel, contrairement aux adventistes. Les convergences entre dénominations mentionnées plus haut trouvent parfois des points de divergence, mais à mon sens pas sur un plan théologique.

33.Selon des modalités qui ne seront pas les mêmes partout (absence de missionnaires occidentaux par exemple) et qui informent à leur tour dans une certaine mesure le contenu du christianisme ainsi transmis (voir Robbins 2004a pour la relation non médiée des Urapmin à la religion chrétienne).

34. Je n'ai pas l'espace suffisant ici pour développer la perspective dont parle Courtney Handman (2014), qui mentionne les phénomènes d'oubli de la conversion, lorsque la domestication du christianisme a été facilitée par la traduction de la Bible en langue vernaculaire (un autre processus d'inculturation/enculturation). Notons que malgré la présence de missionnaires linguistes luthériens dans la vallée de Wonenara pendant 30 ans, personne n'utilise ni ne lit le Nouveau Testament en langue vernaculaire (Bonnemère, 2016 : 214; ce constat fut renforcé par le témoignage d'un professeur de l'école primaire de la vallée, qui me dit un jour ne pas savoir lire sa langue maternelle). 
lée de Wonenara (Papouasie-Nouvelle-Guinée), in Christophe Pons (éd.), Jésus, moi et les autres. La construction collective d'une relation personnelle à Jésus dans les Églises évangéliques: Europe, Océanie, Maghreb, Paris, CNRS Éditions, pp. 227-258.

—, 2016. Church presence and gender relations in the Wonenara valley (Eastern Highlands Province, PNG), TAJA The Australian Journal of Anthropology 27 (2) : Gender Revisited: Towards a re-definition of gender through Christianity in Melanesia, pp. 206-225.

Cannell Fenella, 2006. Introduction: the anthropology of Christianity, in Fenella Cannell (ed.), The Anthropology of Christianity, Durham, NC, Duke University Press, pp. 1-50.

Comaroff John, 2010. The end of anthropology, again: on the future of an in/discipline, American Anthropologist 112, pp. 524-538.

ENGELKe Matthew, 2004. Discontinuity and the Discourse of Conversion, Journal of Religion in Africa 34, 1-2, pp. 82-109.

Godelier Maurice, 1969. La « monnaie de sel » des Baruya de Nouvelle-Guivnée, L'Homme 9 (2), pp. 5-37.

-, 1982. La production des Grands Hommes. Pouvoir et domination masculine chez les $\mathrm{Ba}$ ruya de Nouvelle-Guinée, Paris, Fayard.

—, 2009. Corps, parenté, pouvoir(s) chez les Baruya de Nouvelle-Guinée, in Maurice Godelier et Michel Panoff (éds), Le Corps humain, conçu, supplicié, possédé, cannibalisé, Paris, CNRs éditions, pp. 29-64.

Handman Courtney, 2014. Becoming the Body of Christ. Sacrificing the Speaking Subject in the Making of the Colonial Lutheran Church in New Guinea, Current Anthropology 55, Supp. 10, pp. S205-S215.

Hann Chris, 2007. The anthropology of Christianity per se, European Journal of Sociology 48, pp. 383-410.

Haynes Naomi, 2014. Affordances and Audiences. Finding the Difference Christianity Makes, Current Anthropology 55, S10: The Anthropology of Christianity: Unity, Diversity, New Directions, pp. S357-S365.

Jebens Holger, 2011. Beyond Globalisation and localisation: Denominational Pluralism in a Papua New Guinea Village, The Asia Pacific Journal of Anthropology, Vol.12(1), pp.91-110.
Keller Eva, 2005. The Road to Clarity: Seventh-Day Adventism in Madagascar, New York, NY, Palgrave Macmillan.

Knauft, Bruce M., 2002. Exchanging The Past. A Rainforest World of Before and After. Chicago and London: The University of Chicago Press.

Lemonnier Pierre, 2013. Arcs, flèches et orgue électrique. À propos de modernité et d'offensives évangéliques dans la vallée de Wonenara (Papouasie-Nouvelle-Guinée), in Christophe Pons (éd.), Jésus, moi et les autres. La construction collective d'une relation personnelle à Jésus dans les Églises évangéliques: Europe, Océanie, Maghreb, Paris, CNRs Editions, pp.189-226.

LLOYD Richard, 1973. The Anga language family, in Karl J. Franklin (ed.), The Linguistic situation in the Gulf District and Adjacent Areas, Papua New-Guinea, Canberra, Linguistic circle of Canberra, Pacific Linguistics, Series C, n 26, pp. 33-111.

Malbrancke Anne-Sylvie, 2016a. Une épouse pour de l'argent? Des pratiques matrimoniales en mutation et leurs répercussions socio-culturelles chez les Baruya de Papouasie Nouvelle-Guinée, thèse de doctorat, Paris, EHESS.

—, 2016b. Women don't have testicles: The 'making' of masculinity among twenty-first century Baruya (Eastern Highlands, Papua New Guinea), Paideuma 62, pp. 69-89.

Meyer Birgit, 1998. Make a Complete Break with the past. Memory and Post-Colonial Modernity in Ghanaian Pentecostalist Discourse, Journal of Religion in Africa 28 (3), pp. 316-349.

-, 1999. Translating the Devil: Religion and Modernity among the Ewe in Ghana, Trenton, NJ, Africa World Press.

RÉJAsse Céline, 2011. Vakirà Bara et baptême catholique. Un rituel inculturé à Madagascar, Revue de l'histoire des religions, 1 : 71-92.

Robbins Joel, 1998. Becoming sinners: Christianity and desire among the Urapmin of Papua New Guinea, Ethnology 37, pp. 299-316

—, 2003. On the Paradoxes of Global Pentecostalism and the Perils of Continuity Thinking, Religion 33, 3, pp. 221-31.

-, 2004a. Becoming Sinner: Christianity and Moral Torment in a Papua New Guinea Society, Berkeley \& London, University of California Press. 
-, 2004b. The Globalization of Pentecostal and Charismatic Christianity, Annual Review of Anthropology 33, pp. 117-143.

-, 2005a. Introduction - Humiliation and Transformation: Marshall Sahlins and the Study of Cultural Change in Melanesia, in Joel Robbins and Holly Wardlow (eds.), The Making of Global and Local Modernities in Melanesia, Humiliation, Transformation and the Nature of Cultural Change, Ashgate, University of Pittsburgh, pp.3-21.

—, 2005b. The Humiliations of Sin: Christianity and the Modernization of the Subject among the Urapmin, in Joel Robbins and Holly Wardlow (eds), The Making of Global and Local Modernities in Melanesia, Humiliation, Transformation and the Nature of Cultural Change, Ashgate, University of Pittsburgh, pp. 43-56.

-, 2007a. Continuity Thinking and the Problem of Christian Culture Belief, Time, and the Anthropology of Christianity, Current Anthropology 48 (1), pp. 5-38.

-, 2007b. Between Reproduction and Freedom: Morality, Value, and Radical Cultural Change, Ethnos, 72 (3), pp. 293-314.

—, 2010a. Conversion, hiérarchie et changement culturel : valeur et syncrétisme dans le cadre de l'expansion mondiale du christia- nisme pentecôtiste et charismatique, in Françoise Douaire-Marsaudon et Gabriele Weichart (éds), Les Dynamiques Religieuses dans le Pacifique, Religious Dynamics in the Pacific, Cahiers du CREdo, Pacific-Credo Publications, pp. 235-262.

—, 2010b. Melanesia, Christianity, and cultural change: a comment on Mosko's "Partible Penitents", The Journal of the Royal Anthropological Institute 16 (2), pp. 241-243.

-, 2012. Spirit Women, Church Women, and Passenger Women, Archives de sciences sociales des religions 157 : Christianismes en Océanie, pp. 113-133 (http://assr.revues.org/23646, consulté le 07 août 2016).

—, 2015. Dumont's Hierarchical dynamism. Christianity and individualism revisited, Hau: Journal of Ethnographic Theory 5 (1), pp. 173-195.

SaHLins Marshall, 1981. Historical Metaphors and Mythical Realities: Structure in the Early History of the Sandwich Islands Kingdom, Ann Arbor, ASAO \& University of Michigan Press.

-, 1992. The Economics of Develop-Man in the Pacific, Res 2, pp. 13-25.

Wardlow Holly, 2006. Wayward Women. Sexuality and Agency in a New Guinea Society, Berkeley, University of California Press. 